Monatsschr Kinderheilkd 2014 · 162:978-979

DOI 10.1007/s00112-013-3023-3

Online publiziert: 8. November 2014

๑) Springer-Verlag Berlin Heidelberg 2014
S. Wirth + F. Zepp ${ }^{2}$

${ }^{1}$ Zentrum für Kinder- und Jugendmedizin, HELIOS Klinikum Wuppertal, Universität Witten/Herdecke

${ }^{2}$ Zentrum für Kinder- und Jugendmedizin, Universitätsmedizin Mainz

\title{
Störungen im Bereich des Bewegungsapparats
}

\author{
Herausforderung für Kinder- und \\ Jugendmedizin und Kinderorthopädie
}

Gemäß den Erkenntnissen aus dem Kinder- und Jugendsurvey (KiGGS) des Robert Koch-Instituts sind Störungen im Bereich des Bewegungsapparats häufig. So geben 13\% der untersuchten Kinder und Jugendlichen Rückenschmerzen an, und 5\% haben eine Skoliose [1]. Klinischer Alltag sind Haltungsschäden oder funktionelle Störungen, die bei anhaltenden Beschwerden neben der Untersuchung durch den Kinder- und Jugendarzt auch einer Beurteilung durch einen Kinderorthopäden bedürfen. Die Kooperation zwischen der Kinder- und Jugendmedizin und der Kinderorthopädie hat eine erfreulich lange Tradition. Weitere Herausforderungen werden im Kontext der beunruhigenden Prävalenzzunahme von Übergewicht und Adipositas um 50\% für beide Disziplinen aktuell. Gerade Übergewicht belastet den Bewegungsapparat über die Jahre in besonderem Maß [2].

Auch wenn die meisten orthopädischen Therapiekonzepte für Kinder konservativ sind, erfordern einige akute und chronische Erkrankungen gelegentlich die rasche operative Intervention. Schmerzsymptome, insbesondere im Bereich der Extremitäten, müssen ernst genommen und kompetent diagnostiziert werden. Eine gute interdisziplinäre Abstimmung und Kooperation zwischen Kinderorthopäden und Kinder- und Jugendärzten legt die Grundlage für die optimale Behandlung der betroffenen Patienten.

\section{D) Gute interdisziplinäre Abstimmung legt Grundlage für die optimale Behandlung}

Das Schwerpunktthema in diesem Monat beschäftigt sich mit 4 interessanten Teilgebieten der Kinderorthopädie. Zwei Arbeiten behandeln schwerwiegende Störungen der Hüftentwicklung. Ein weiterer Beitrag fasst die aktuellen Erkenntnisse zu seltenen kartilaginären Exostosen zusammen, und der 4. Beitrag thematisiert Patienten mit spastischen Gangstörungen.

Manig hat die aktuellen diagnostischen und therapeutischen Prinzipien zum M. Perthes zusammengestellt. Das Krankheitsbild ist seit Langem bekannt und betrifft eines von 10.000 Kindern. Das ist zwar nicht besonders häufig, aber auch nicht so selten, dass man im klinischen Alltag diese (Differenzial-)Diagnose vernachlässigen darf. Der Erkrankungsgipfel des M. Perthes liegt im Vorschul- und Grundschulalter. Daher muss bei Hüft- und Knieschmerzen in dieser Altersgruppe immer auch an eine Nekrose des Femurkopfes gedacht werden. Die therapeutische Intervention zu einem möglichst frühen Zeitpunkt ist besonders aussichtsreich.

Das Gleiche gilt für die häufigste Hüfterkrankung des Heranwachsenden, die Epiphyseolysis capitis femoris (ECF). Zilkens et al. präsentieren eine aktuelle Über- 
sicht, in der deutlich wird, dass der Diagnosezeitpunkt auch bei dieser Erkrankung prognostisch besonders wichtig ist. Die rechtzeitige adäquate Behandlung kann schwerwiegende Langzeitkomplikationen vermeiden. In diesem Zusammenhang soll auf die viel zitierte Erkenntnis „Schmerz im Knie - vergiss die Hüfte nie“ hingewiesen werden.

Eine zugegebenermaßen seltene Krankheitsentität stellen die multiplen kartilaginären Exostosen dar. Umso interessanter ist die Zusammenfassung von Rupprecht et al., die dieses autosomal-dominant vererbte Krankheitsbild darstellt. Das Langzeitmanagement orientiert sich konsequent am Beschwerdebild und soll umsichtig erfolgen. Besondere Erfahrung des spezialisierten Kinderorthopäden ist dazu erforderlich. Eine operative Intervention bedarf der sorgfältigen Indikationsstellung und muss mit den Betroffenen/Eltern ausführlich erörtert werden.

Die optimale medizinische Betreuung von Kindern und Jugendlichen mit spastischen Bewegungsstörungen ist eine besondere Herausforderung. Gerade im Hinblick auf den skeletalen Wachstumsprozess müssen diese Patienten mit speziellen biomechanischen diagnostischen Maßnahmen begleitet werden. Brunner erläutert in seinem Beitrag die Bedeutung der äußerst differenzierten Diagnostik mit dem Ziel, die für das Individuum optimalen therapeutischen Möglichkeiten und Therapieziele adäquat zu definieren. Der Einsatz von Orthesen soll sorgfältig abgewogen indiziert erfolgen. Beurteilung von Muskelfunktion und Stabilität im Standbein haben ebenfalls wichtige Bedeutungen, da sich an den Ergebnissen der Einsatz der konservativen und operativen Maßnahmen ausrichten lässt.

Zusammenfassend illustrieren die Beiträge dieser Monatsschrift Kinderheilkun$d e$, wie hochkomplex Diagnostik und Therapie in der Kinderorthopädie entwickelt sowie welche differenzierten Maßnahmen möglich und notwendig sind, um eine individuelle, auf den Patienten optimal abgestimmte Behandlung zu ermöglichen. Führendes Ziel ist es, Langzeitkomplikationen zu vermeiden und/oder die Lebensqualität und Teilhabe der Kinder- und Jugendlichen mit Einschränkungen ihrer Motilität zu verbessern. Kennt- nis auf diesem orthopädischen Teilgebiet sind für den betreuenden Kinder- und Jugendarzt wichtig, kommt ihm in der Lotsenfunktion doch eine besondere Verantwortung zu.

Wir freuen uns, mit diesen Beiträgen einen aktuellen Überblick über wichtige Themen der Kinderorthopädie geben zu können. Wir danken den Autoren für ihr Engagement und wünschen Ihnen beim Lesen viel Freude in der Hoffnung, dass die Informationen für Sie im klinischen Alltag nutzbringend sind.

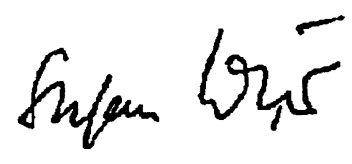

Prof. Dr. S. Wirth



Prof. Dr. F. Zepp

\section{Korrespondenzadresse}

\section{Prof. Dr. S. Wirth}

Zentrum für Kinder- und Jugendmedizin, HELIOS Klinikum Wuppertal Universität Witten/Herdecke Heusnerstr. 40, 42283 Wuppertal stefan.wirth@helios-kliniken.de

\section{Einhaltung ethischer Richtlinien}

Interessenkonflikt. S. Wirth und F. Zepp geben an, dass kein Interessenkonflikt besteht.

\section{Literatur}

1. Kamtsiuris P, Atzpodien K, Ellert U, Schlaud M (2007) Prävalenz von somatischen Erkrankungen bei Kindern und Jugendlichen in Deutschland. Bundesgesundheitsblatt Gesundheitsforschung Gesundheitsschutz 50:686-700

2. Hölling H, Schlack R, Kamtsiuris P et al (2012) Die KiGGS-Studie. Bundesgesundheitsblatt Gesundheitsforschung Gesundheitsschutz 55:836-842

\section{Ausschreibung Hermann-Emminghaus-Preis 2015}

Gesucht: Exzellente Arbeiten im Fach „Kinder- und Jugendpsychiatrie und -psychotherapie"

Der Hermann-Emminghaus-Preis ist der älteste kinder- und jugend-psychiatrische Forschungspreis im deutschsprachigen Raum. Er richtet sich an Wissenschaftler, die empirische Forschung auf dem Gebiet der Kinder- und Jugendpsychiatrie und -psychotherapie, insbesondere der biologischen Kinder-und Jugendpsychiatrie, betreiben und in der Regel nicht länger als zehn Jahre im Fach wissenschaftlich tätig sind. Es können ausschließlich Arbeiten eingereicht werden, die noch nicht anderweitig ausgezeichnet worden sind.

Psychodynamisch, genetisch oder zerebralorganisch orientierte Forschung kommt ebenso für eine Bewerbung in Betracht wie epidemiologische, katamnestische oder therapeutische Studien. Die Arbeit ist in deutscher oder englischer Sprache einzureichen.

Die Verleihung des Hermann-Emminghaus-Preises 2015 wird Anfang März 2015 im Rahmen des XXXIV. Kongresses der Deutschen Gesellschaft für Kinder-und Jugendpsychiatrie, Psychosomatik und Psychotherapie e. V. (DGKJP) in München stattfinden.

Mit der letzten Auszeichnung im Jahr 2013 wurde der Kinder- und Jugendpsychiater PD Dr. med. Timo Vloet vom Universitätsklinikum Aachen in Anerkennung seiner wissenschaftlichen Arbeiten zum Thema "Neurobiologische Aspekte dissozialer Störungen" geehrt.

Weitere Informationen zum HermannEmminghaus-Preis und zu den Teilnahmebedingungen sind unter www.emminghaus-preis.de zu finden.

Quelle: Kuratorium des Hermann-Emminghaus-Preises vertreten durch Prof. Dr. med. Dr. rer. nat. Martin H. Schmidt, martin.schmidt@zi-mannheim.de 